\title{
Acknowledgment
}

The Editor acknowledges the assistance of the following persons who served as ad hoc reviewers for Psychology of Violence during the past year.

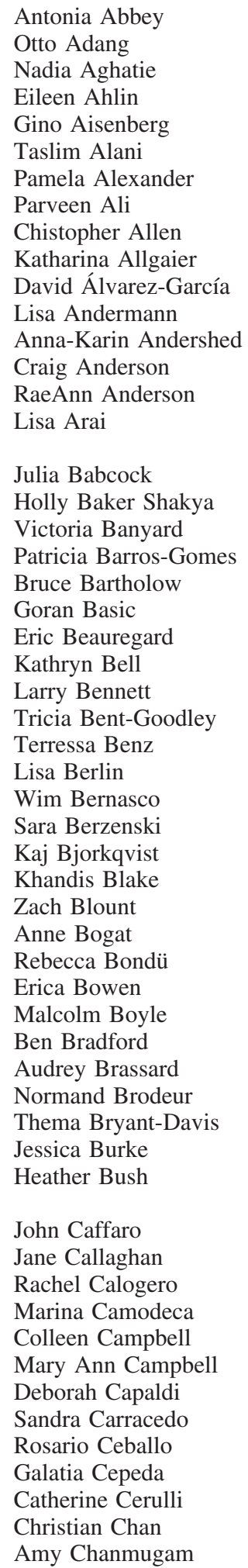

John Chapin
May Chen
Hye Jeong Choi
Gina Chowa
Andrea Cimino
Stephen Clipper
Ann Coker
Delphine Collin-Vézina
Jonathan Comer
Jennifer Copp
Cory Crane
Kimberly Crossman
Rebecca Cudmore
Matthew Cuellar
Dean Dabney
Christina Dardis
M. Davidson
Myrna Dawson
Joaquin de Paul
Jacqueline De Puy
Dana DeHart
Thomas Denson
Sarah Desmarais
David DiLillo
Karsten Donnay
Emily Douglas
Laura Dryjanska
Ed Dunbar
Emily Dworkin
Christopher Eckhardt
Kale Edmiston
Miriam Ehrensaft
Hedwig Eisenbarth
Manuel Eisner
Chris Elledge
Dorothy Espelage
Deinera Exner-Cortens
Jennifer Fanning
Naomi Farber
Kazi Nazrul Fattah
Angèle Fauchier
Gene Feder
Lisa Fellin
Rachel Fenton
Christopher Ferguson
Paula Fite
Esther Flanagan
Julianne Flanagan
Diane Follingstad
Heather Foran
Anjali Forber-Pratt
Dianne Ford
Julian Ford
David Forde
Gregory Fosco

Cindy Fraga Rizo
Joseph Franklin
Jennifer Freyd
Brett Gardner
Carlo Garofalo
Jacinta Gau
Christine Gidycz
Amanda Gilmore
Andrew Gladfelter
Elizabeth Goncy
Lisa Goodman
Simon Goodman
Bradley Goodnight
Laurie Graham
Sandra Graham-Bermann
Jennifer Greif Green
Paula Grubb
John Grych
Steve Guglielmo
Lauren Gulbas
Jhumka Gupta
Elizabeth Gurian

Ute Habel

Michelle Haikalis

Abigail Hatcher

Samuel Hawes

David Hayes

Denise Haynie

Tracy Hipp

Jericho Hockett

George Holden

E. Alison Holman

Melissa Holt

Jun Sung Hong

Sylco Hoppenbrouwers

Michael Hughes

Patricia Hynes

Katherine Iverson

James Ivory

Angela Jacques-Tiura

Anna Jaffe

Dawn Johnson

Wendi Johnson

Marcus Juodis

Rachel Kahn

Kimberly Kamper-DeMarco

Gery Karantzas

Erika Kelley

Angie Kennedy

Traci Kennedy

Justin Kern

Mary Kernic

Roxanne Khan
Hyoun Kim

Katherine Kimble

Michael King

Wendy Kliewer

Elias Kourkoutas

Sherianne Kramer

Zlatan Krizan

MJJ Kunst

Gabriel Kuperminc

Betty Kwagala

Lareina La Flair

Susan Lagdon

Sharon Lambert

Malose Langa

Jennifer LanghinrichsenRohling

Paul Lanier

Stephen Lawoko

Monique LeBlanc

Amanda LeCouteur

Jonathan Lee

Stephen Leff

Amy Lehrner

Deborah Levesque

Marie Lindegaard

Taryn Lindhorst

Alan Litrownik

Heather Littleton

T. K. Logan

Brenda Lohman

Elise Lopez

Beatriz Lucas-Molina

Amy Lyndon

Megan Maas

Harriet MacMillan

Christopher Magee

Eric Mankowski

Amy Marshall

Rainier Masa

Christopher Maxwell

David Mazeika

Melanie McCarry

Courtney McDonald

Maureen McHugh

Blake McKimmie

Krista Mehari

Annelise Mennicke

Melissa Merrick

Gabriel Merrin

Joshua Miller

Laura Miller-Graff

Kimberly Mitchell

Pegah Moghaddam

Oswaldo Montoya

Ton Mooij

Penelope Morrison 
Carrie Mulford

Tine Mundbjerg Eriksen

Christopher Murphy

Angela Narayan
Angela Neal
Jennifer Neal
Andrew Newton
Laura Niemi
Phyllis Niolon
Nkiru Nnawulezi
Raymond Novaco
Lindsay Oberleitner
Ciarán O'Driscoll
Ijeoma Ogbonnaya
Megan Oka
Emily Olsen
Mark Olver
Linda O’Neill
Susan Opotow
Lindsay Orchowski
Pamela Orpinas
Gina Owens
Tom Page
Tom Pakkanen
Elizabeth Parker
Michele Parkhill
Kathleen Parks
Cynthia Pearson
William Pedersen
Debra Pepler
Nathan Perkins
Zoe Peterson
Jonathan Pettigrew

Afroditi Pina

Caroline Piotrowski

Richard Pond

Esther Price

Jennifer Price-Wolf

Brian Quigley

Dakari Quimby

Daniella Rafaely

Shireen Rajaram

Lynette Renner

Callie Rennison

Tyson Reuter

Edward Reynolds

Deborah Richardson

Rebecca Rodriguez

Katherine Roof

Alvina Rosales

Roderick Rose

Emily Rothman

Linda Rouse

Linda Rubin

Lynnmarie Sardinha

Don Saucier

Nichole Scaglione

Marya Schulte

Meghan Shanahan

Jessica Shaw

Andrew Sherrill

Ryan Shorey

Joann Shortt

Lorelei Simpson Rowe

Lauren Sippel

Karen Slade
Allison Smith

Christine Smith

Stephanie Smith

Susan Sorenson

Rebecca Spizzirri

Trevor Spratt

Nicky Stanley

Cynthia Stappenbeck

Deborah Stearns

Garth Stevens

Sandra Stith

Carla Stover

David Sugarman

Kevin Swartout

Casey Taft

Elizabeth Taylor

Julie Taylor

Jeff Temple

Maria Testa

Cynthia Thomsen

Brandy Tiernan

Patti Timmons Fritz

Robert Tokunaga

Jemma Tosh

Elisa Trucco

Brianna Turner

Heather Turner

Sarah Ullman

Kevin Vagi

Rosa Valls

Marco van Bommel

Solveig Karin Bø Vatnar

Noah Venables
Judith Verweijen

Michael Vitacco

Irene Vitoroulis

Kate Walsh

Wendy Walsh

Mark Walters

Mikel Walters

L. Monique Ward

Laura Watkins

Kristina Webber

Rhiana Wegner

Christine Wekerle

Rebecca Weston

Stuart White

Sarah Whitton

Damien Williams

Tiara Willie

Laura Wilson

Milo Wilson

Sylia Wilson

Jacqueline Woerner

Jennifer Wong

Peggilee Wupperman

Matthew Yalch

Kathryn Yount

Nicole Yuan

Amie Zarling

April Zeoli

Eileen Zurbriggen

Izabela Zych

\section{Editors of the Year}

\section{Consulting Editor of the Year 2017: Kevin Swartout}

Dr. Kevin Swartout is an Associate Professor of Psychology and Public Health at Georgia State University. His expertise is in the epidemiology and social correlates of violence and victimization, as well as measurement and data analysis issues related to violence research.

\section{Contributing Editor of the Year 2017: Cynthia Stappenbeck}

Dr. Cynthia Stappenbeck is an Assistant Professor in the Department of Psychiatry and Behavioral Sciences at the University of Washington. Her expertise is in the effects of alcohol use on sexual assault victimization and interpersonal violence perpetration, and the co-occurrence of alcohol use and PTSD. 